\title{
Evidence for phonetic processing of cues to place of articulation: Perceived manner affects perceived place
}

\author{
GUY CARDEN and ANDREA LEVITT \\ Haskins Laboratories, New Haven, Connecticut 06510 \\ and \\ PETER W. JUSCZYK and AMANDA WALLEY \\ Dalhousie University, Halifax, Nova Scotia, Canada $B 3 H 4 H 8$
}

\begin{abstract}
Using stimuli that could be labeled either as stops $[\mathrm{b}, \mathrm{d}]$ or as fricatives $[\mathrm{f}, \mathrm{v}, \theta, \delta]$, we found that, for a given acoustic stimulus, perceived place of articulation was dependent on perceived manner. This effect appeared for modified natural syllables with a free-identification task and for a synthetic transition continuum with a forced-choice identification task. Since perceived place could be changed by changing manner labels with no change in the acoustic stimulus, it follows that the processing of the place feature depends on the value the listener assigns to the manner feature rather than directly on any of the acoustic cues to manner. We interpret these results as evidence that the identification of place of articulation involves phonetic processing and could not be purely auditory.
\end{abstract}

It is clear that a model for speech perception will include general-purpose auditory mechanisms at the input end of the system and speech-specific phonetic or phonological mechanisms at the output end where segments are identified. The interesting theoretical question is, how far in the process can we go using mechanisms that are also used in nonspeech processing? If we assume, for example, that the listener proceeds by identifying such features as place or manner and then combining these features into segments, it is clear that the combination operation must include mechanisms that are specialized for speech processing ("phonetic mechanisms"). In principle, however, the mechanisms involved in processing the cues to the features might be purely auditory; and, in fact, whether phonetic mechanisms are needed to process the feature cues remains a matter of controversy. See, for example, Liberman and Studdert-Kennedy (1978) and Stevens and Blumstein (1978) for some references to the extensive literature on these questions.

This research was supported in part by NICHD Grant HD 01994 and BRS Grant 05596 to Haskins Laboratories, and in part by N.S.E.R.C. Grant A-0282 to Peter W. Jusczyk. We are grateful to Terry Halwes, Alvin Liberman, Rod McGuire, David Pisoni, Bruno Repp, Linda B. Smith, and Michael Studdert-Kennedy for useful advice and criticisms, though of course none of them necessarily agrees with us. We are also grateful to Janice Murray, Christopher Murphy, and Leon Serafim for assistance in running subjects. G.C. was affiliated with Yale University at the time of the study. A.L. is also af filiated with Wellesley College; G.C., P.W.J., and A.W. are now affiliated with the University of British Columbia, the University of Oregon, and Indiana University, respectively. Correspondence should be sent to Andrea Levitt, Haskins Laboratories, 270 Crown Street, New Haven, Connecticut 06510.
It is hard to get a clear test of this issue, since, in most experiments, the effects that seem to imply phonetic processing might imaginably be caused by complex auditory interactions (see, for example, the discussion in Pisoni, 1980). What we need is an experimental design that controls for auditory interactions. The best possible control is to hold the acoustic stimulus constant: If we can find a manipulation in which the effect of interest can be triggered by a change in percept alone, then, in principle, no purely auditory explanation would be possible, and we would have to infer that phonetic processing was involved.

A series of experiments have succeeded in doing this by constructing stimuli that could be perceived either as speech or as nonspeech, or as both simultaneously. Two different techniques have been used, pure-tone synthesis (Dorman, 1980; Bailey, Summerfield, \& Dorman, Note 1); Best, Morrongielo, \& Robson, Note 2) and duplex perception (Isenberg \& Liberman, 1978; Liberman, 1980). In each study, the authors investigated the familiar formant-transition cue to place of articulation. They manipulated the experimental conditions so that a given acoustic stimulus was perceived as speech in one condition and as nonspeech in another; finding differences in the way the stimuli were perceived in the speech and nonspeech conditions (e.g., different discrimination functions), they inferred that the subjects were using special-purpose phonetic processing in the speech condition.

These experiments provided evidence for phonetic processing by showing that a given acoustic stimulus was processed differently in speech and nonspeech 
conditions. As Pisoni (1980) points out, it is possible to argue that the speech/nonspeech contrasts in these experiments result from the fact that the subjects are familiar with speech signals but not with the nonspeech analogs, so that they find it easier to selectively attend to the relevant cues in the speech condition than to those in the nonspeech condition. We could therefore get a sharper test for phonetic processing if we compared two different classes of speech stimuli rather than speech and nonspeech. How could we do this?

One method is to look for a case in which a given acoustic stimulus is processed differently under two phonetic conditions. The stimulus involved cannot merely be ambiguous; we need to find cases in which one phonetic dimension is dependent on a second and then to manipulate the percept for that second dimension without changing the acoustic stimulus. The familiar dependencies among phonetic features offer a promising place to start. For example, Eimas, Tartter, Miller, and Keuthen (1978), Miller (1977), and Smith (1973) found evidence that in a number of cases the identification of place of articulation (e.g., labial vs. dental) was dependent on manner (e.g., stop vs. nasal or stop vs. fricative). In these studies, the manner dimension was cued acoustically: For example, Miller (1977) constructed synthetic voiced stop and nasal continua in which the place feature was cued by variations in the transitions for F2 and F3 and the stop/nasal manner difference was cued by adding a nasal resonance and flattening the initial transition of $F 1$ in the nasal stimuli. Miller found small, but significant, differences in the labial vs. dental boundary for the two continua. From our point of view, the interesting question is, does this boundary shift reflect phonetic processing or only auditory interactions between the acoustic cues to manner and place?

The English stops and fricatives in the labial-todental' range look like a good choice for a study investigating whether changes in perceived manner will alter perception of place-of-articulation. Eimas et al. (1978) report a mutual, but asymmetrical, dependency in processing place and manner information for [ba, $\mathrm{da}, \mathrm{va}, \mathrm{za}$, in which place is more dependent on manner than manner is on place. Moreover, we can find a parallel dependency in production if we select the right stops and fricatives to compare: For example, in the labial range, the place of articulation for stops $[p, b]$ is bilabial, while the place of articulation for the fricatives $[f, v]$ is labiodental. Similarly, in the dental range, the stops $[t, d]$ are alveolar, while the fricatives $[\theta, \delta]$ are interdental. Thus, for English stops and fricatives, the dependencies observed for the perception of place and manner information seem to be mirrored, at least in part, by the presence of similar dependencies in production. If we look at the set $[\mathrm{b}, \mathrm{f}, \mathrm{v}, \mathrm{d}, \theta, \delta],{ }^{2} \mathrm{a}$ change in the manner feature will require a change in the place feature in articulation: The series runs from bilabial $[b]$ to labiodental $[f, v]$ to interdental $[\theta, \partial]$ to alveolar [d]. This set has the further advantage that we can focus on a single cue to place, the format transitions, which will be a sufficient cue to place both for the stops and for this set of fricatives. Harris (1958) gives evidence that the transitions are the primary cue distinguishing $[f, v]$ from $[\theta, \widetilde{\gamma}]$ and that there is little, if any, place information in the frication noise, contrasting with such fricative comparisons as [s] vs. $\left[\int\right]$, in which the noise is the major place cue.

Let us assume, then, that the labial-to-dental changes in articulation in the stop-fricative series $[b, f, v, d, \theta, \delta]$ correspond to formant transition changes that can be arranged along a single continuum. The simplest auditory hypothesis would predict that the place boundary corresponds to a psychophysical boundary. Given a single continuum, there would be no reason to expect that different psychophysical boundaries would appear, depending on the manner, except to the extent that the acoustic cues 10 manner interacted auditorily or psychophysically with perception of the transition (cf. the nonspeech results of Pisoni, 1980). If we were to find different boundaries lor the stops and the fricatives along the same continuum, and the difference could not be attributed to an auditory or psychophysical interaction, then the difference between the boundaries must reflect phonetic processing. How could we show that the difference was not due to an auditory or psychophysical interaction? Recall that our first step was to establish a dependency, in this case a place boundary shift that depended upon the acoustically cued manner of the stimulus. Given this sort of dependency, what would happen if we changed only the perceived manner without changing the acoustic stimulus? Would we find the same dependency we found when the change in manner was cued acoustically? A negative result would not prove much, since it is natural to suppose that some feature dependencies may involve only auditory interactions and, in any case, it would be difficult to prove that phonetic processing was not involved; but a positive result would be strong evidence. If, for example, we could shift a place boundary simply by changing perceived manner, phonetic processing would have to be involved; since the stimuli in the two conditions would be acoustically identical, in principle no purely auditory explanation would be possible.

Our objective in the following series of experiments was to investigate manner/place interactions in the set of stops and fricatives $[b, f, v, d, \theta, \delta]$. We needed first to establish whether there was a manner/place dependency, which would be expected given the earlier work on feature dependencies with similar sets. If we were to find such a dependency, we would need to change the perceived manner without changing the acoustic stimulus and to test whether the dependency was the same as that found when the manner change was acoustically cued. We could do this in two ways: (1) We could construct stimuli that were ambiguous in manner, either by synthesis or by modifying naturally produced 
syllables. If we were to give the subjects a free-choice identification task, they would sometimes identify a given ambiguous stimulus as a stop and sometimes as a fricative. We could then observe whether this difference in perceived manner interacted with place identifications. We used this method in Experiment 1. (2) We could use a forced-choice identification task and change the instructions so that in one condition the subject was required to label a given set of stimuli as stops and in the other condition was required to label the same stimuli as fricatives. Again, we could observe whether the labeling interacted with place identifications. We used this method in Experiment 2.

\section{EXPERIMENT 1}

The purpose of Experiment 1 was to investigate the perception of the formant-transition cue to place of articulation in naturally produced syllables and, in particular, to see whether the perception of the place cue was affected by a change in perceived manner (stop vs. fricative). We therefore wanted to create stimuli that were ambiguous in perceived manner without changing the naturally produced formant transitions.

The primary cue to the stop/fricative manner contrast in CV syllables is the duration of the aperiodic noise that precedes the formant transitions. Fricatives have a relatively long noise (typically $100-200 \mathrm{msec}$ ), a "frication"; stops have a short noise (typically a few milliseconds), a "burst." Our preliminary work had shown that, if one removes the burst or frication, the resulting truncated syllable is most often heard as a (voiced) stop but is somewhat ambiguous between stop and fricative manner. Cutting off the frication from fricatives, therefore, seemed to be a promising way of producing the ambiguous stimuli we needed.

We, therefore, expected that the truncated fricatives would be perceived as ambiguous in manner, and we wanted to see whether the perceived place was different for the two perceived manners. We exploited this ambiguity by giving the subjects a relatively natural free-choice identification task and simply examining the resulting pattern of perceived place for a given perceived manner. Our preliminary results had shown that the truncated labiodental and interdental fricatives were most often identified as $[f, v, b, \theta, \delta, d]$, so we used natural and truncated versions of these six syllables.

\section{Method}

Stimuli. Pretests had shown that the labiodentals $[f, v]$ gave reasonably consistent patterns of identification across speakers, but that there was substantial variation for the interdentals $[\theta, \delta]$. We used these pretest results to select four adult male speakers who represented the observed range of variation. P.N. and P.B. are British; T.H. and D.I. are Americans.

For each speaker, we recorded three tokens of the $\mathrm{CV}$ syllables [fa], [va], [ba], [ $\theta \mathrm{a}$ ], [ $\gamma_{\mathrm{a}}$ ], and [da], using an Ampex AG500 tape recorder. Each loken was digitized and low-pass filtered at $4.9 \mathrm{kHz}$ using the Haskins Laboratories pulse code modulation (PCM) system (Cooper \& Mattingly, 1969). We then used the PCM system to delete the bursts or frications from the digitized natural syllables, cutting at the point of zero or near-zero amplitude nearest to the end of the burst or frication. ${ }^{3} \mathrm{We}$ will call the resulting truncated

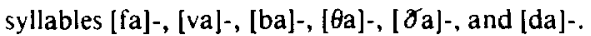

Separate audio tapes were prepared for each speaker's tokens. Each tape consisted of 10 repetitions of each of the three tokens of the 6 natural syllables and 10 repetitions of the truncated versions of these 18 syllables. These 360 stimuli were arranged in a random order with a $3-\mathrm{sec}$ ISI and an additional $4 \mathrm{sec}$ after every 10th stimulus. The same randomization was used for all four tapes.

Subjects. Twelve college-age native speakers of English were paid for participating in two experimental sessions. None reported any history of speech or hearing disorder.

Procedure. The subjects were tested in small groups in a quiet room; the stimulus tapes were presented binaurally on an Ampex AG500 tape recorder through Grason-Stadler TDH 39-300Z headphones at a comfortable level. The subjects heard two tapes in the first session, and the other two in a second session a day or two later. The order of presentation was counterbalanced across subjects.

The subjects were told that the stimuli represented English consonant-vowel syllables, and that their job was to identify the initial consonant in each syllable by writing the appropriate letter on their answer sheets. Since $[\theta]$ and $[\partial]$ are represented by the same English letters "th," the subjects were told to use "th" for $[\theta]$ and "dh" for [ø]; they practiced with minimal pairs such as "thigh" [8ay] and "thy" [øay] until they reported that they understood the task. The instructions emphasized that all the stimuli began with an English consonant and that, if any stimulus sounded strange or ambiguous, the subject should write down the English consonant that sounded closest to what he heard. The subjects were, however, given the option of writing "none" if they heard no consonant at all.

\section{Results}

Table 1 gives the results; for each syllable type, the responses are pooled for the four speakers and 12 subjects. Not surprisingly, the results for the truncated syllables are considerably more variable than were those for the natural syllables. The complex pattern is easier to interpret if we look at place and manner separately.

When a subject identifies a stimulus as beginning with a particular segment " $b$ " or " $f$ " or " $\theta$ " or " $d$,"

Table 1

Percentages of Responses for Natural and Truncated Syllables (Experiment 1)

\begin{tabular}{|c|c|c|c|c|c|c|c|c|c|c|}
\hline \multirow{3}{*}{$\begin{array}{l}\text { Stim- } \\
\text { ulus }\end{array}$} & \multicolumn{8}{|c|}{ Responses } & \multirow[b]{3}{*}{$\mathbf{N}$} & \multirow[b]{3}{*}{0} \\
\hline & \multicolumn{4}{|c|}{ Labial } & \multicolumn{4}{|c|}{ Dental } & & \\
\hline & $\mathrm{p}$ & $\mathrm{b}$ & f & $v$ & $\mathrm{t}$ & d & $\theta$ & $\gamma$ & & \\
\hline $\begin{array}{l}{[\mathrm{ba}]} \\
{[\mathrm{ba}]-}\end{array}$ & & $\begin{array}{l}90 \\
59\end{array}$ & $\begin{array}{l}1 \\
6\end{array}$ & $\begin{array}{l}6 \\
3\end{array}$ & & & & $\begin{array}{l}1 \\
1\end{array}$ & $\begin{array}{r}1 \\
31\end{array}$ & \\
\hline $\begin{array}{l}{[\mathrm{fa}]} \\
{[\mathrm{fa}]-}\end{array}$ & 1 & 49 & $\begin{array}{l}80 \\
18\end{array}$ & $\begin{array}{l}3 \\
1\end{array}$ & & & $\begin{array}{r}14 \\
1\end{array}$ & $\begin{array}{l}1 \\
1\end{array}$ & 29 & \\
\hline $\begin{array}{l}{[\mathrm{va}]} \\
{[\mathrm{va}]-}\end{array}$ & & $\begin{array}{r}1 \\
68\end{array}$ & 4 & $\begin{array}{l}79 \\
20\end{array}$ & & & $\begin{array}{l}3 \\
1\end{array}$ & $\begin{array}{r}16 \\
2\end{array}$ & 5 & \\
\hline $\begin{array}{l}{[\mathrm{da}]} \\
{[\mathrm{da}]-}\end{array}$ & & 1 & & & & $\begin{array}{l}99 \\
76\end{array}$ & 3 & 6 & 6 & 8 \\
\hline $\begin{array}{l}{[\theta \mathrm{a}]} \\
{[\theta \mathrm{a}]-}\end{array}$ & & 27 & $\begin{array}{r}16 \\
4\end{array}$ & 2 & & 9 & $\begin{array}{l}80 \\
20\end{array}$ & $\begin{array}{r}3 \\
11\end{array}$ & 25 & 2 \\
\hline $\begin{array}{l}{[\delta a]} \\
{[\delta a]-}\end{array}$ & & 9 & & 1 & & 37 & $\begin{array}{l}18 \\
10\end{array}$ & $\begin{array}{l}81 \\
30\end{array}$ & 8 & 4 \\
\hline
\end{tabular}

Note $-N=$ none; $O=$ other. 
he implicitly makes both a place judgment (labial vs. dental) and a manner judgment (stop vs. fricative). Recall that the purpose of the truncation was to create stimuli that were ambiguous between stop and fricative manner. This manipulation was reasonably successful: Overall, the truncated fricative syllables were identified as beginning with labial or dental stops $50 \%$ of the time, with labial or dental fricatives $[f, v, \theta, \delta] 31 \%$ of the time, with other initial consonants $1 \%$ of the time, and with no initial consonant at all $17 \%$ of the time.

We now need to look for dependencies between the place and manner judgments implied by the segmental identifications. Table 2 gives a conditional probability chart for each truncated fricative, showing the proportion of labial and dental responses when that syllable was identified as a stop compared with the proportion when it was identified as a fricative. All four syllable types show an apparent dependency between perceived place and perceived manner: When a truncated fricative is identified as a fricative, the labial/dental proportion is essentially the same as for the original untruncated fricative; when the truncated fricative is identified as a stop, the proportion of labial responses increases. The increase is relatively small for [fa]- and [va]-, slightly larger for [8a]-, and very large for $[\theta \mathrm{a}]-$, for which the labial/dental proportion is almost reversed for the two perceived manners. If we look at the data pooled across subjects, speakers, ${ }^{4}$ and tokens, the increase is significant for all four syllable types, $\mathrm{p}<.01$ by chi-square. The pooled data in Table 2 represent a summation of cases in which acoustically identical stimuli were perceived sometimes as stops and sometimes as fricatives, with more labial identifications when the syllable was perceived as a stop. ${ }^{5}$

A test on the pooled data, however, is not really appropriate, since an apparently significant result might be obtained by pooling data from a group of subjects, speakers, or tokens with consistently labial stop responses with data from another group with consistently dental fricative responses, even though no individual showed the place/manner dependency in which we are interested. We can test this by looking at the

Table 2

Percentage of Labial and Dental Responses, Given Perceived Mamner (Stop vs. Fricative) (Experiment 1)

\begin{tabular}{clcc} 
& & \multicolumn{2}{c}{ Response } \\
\cline { 3 - 4 } Stimulus & Manner & Labial & Dental \\
\hline \multirow{2}{*}{ fa] -} & Stop & 99 & 1 \\
& Fricative & 91 & 9 \\
{$[\mathrm{va}]-$} & Stop & 100 & \\
& Fricative & 88 & 12 \\
{$[\theta$ a $]-$} & Stop & 75 & 25 \\
& Fricative & 16 & 84 \\
{$[$ (6a]- } & Stop & 20 & 80 \\
& Fricative & 3 & 97 \\
\hline
\end{tabular}

response patterns from the 12 subjects independently, using chi-square, or, when the distribution made chisquare inappropriate, a Fishers exact test. For $[\theta a]-$, the shift is significant for 10 of the 12 subjects, $p<.025$; for $[8 \mathrm{a}]-$, the shift is significant for 8 out of the $12 \mathrm{sub}$ jects, $p<.05$; and for [fa]- and [va]-, the shift is significant at $p<.05$ for only 3 of the 12 subjects.

\section{Discussion}

Experiment 1 supports earlier studies that found manner/place dependencies; it extends those studies in the limited sense that the dependency we found involves the interdentals $[\theta, \delta]$.

The interesting feature of this dependency is that perceived place varies with changes in perceived manner even when the stimuli are acoustically identical. It follows that this dependency cannot be caused by any of the acoustic cues to manner or place, but must instead be caused by the subject's (necessarily phonetic) manner or place decision.

The contrast between [ $\theta \mathrm{a}]$ - and the other truncated fricatives that we see in Table 2 suggests that there may well be two distinct mechanisms involved, one that applies to all the truncated fricatives and produces a relatively small effect and one that applies only, or most strongly, to $[\theta \mathrm{a}]-$ and produces a more substantial shift in the same direction. In the following experiment, we will focus on the stronger effect that we found with $[\theta \mathrm{a}]-;$ we will discuss the other syllables briefly in the General Discussion. Because the task in Experiment 1- was a free identification, we had no evidence as to whether the place/manner dependency was mutual or asymmetric, with one feature controlling the other. Previous work on place/manner dependencies (Eimas et al., 1978; Miller, 1977) had suggested that we were likely to find a mutual, but asymmetric, dependency, with place more dependent on manner than vice versa. The most important result of Experiment.1 was the observation that there was a dependency between perceived place of articulation and perceived manner. The purpose of Experiment 2 was to replicate and explore this result under more tightly controlled conditions.

\section{EXPERIMENT 2}

We used synthetic stimuli and both identification and discrimination tasks; we manipulated perceived manner by changing the labels for the stimuli in a forced-choice identification task.

\section{Method}

Subjects. The subjects were 20 college-age native speakers of English from Dalhousie University. Each subject was paid \$4 for participating in two experimental sessions on succesive days. None of them had had substantial previous experience with synthetic speech.

Stimuli. The stimuli were constructed using the OVE IIlc serial synthesizer. We synthesized two nine-item continua: a [ba]-to- 
Table 3

Starting Formant Frequencies for F2 and F3 (Experiment 2)

\begin{tabular}{ccc}
\hline $\begin{array}{c}\text { Stimulus } \\
\text { Number }\end{array}$ & F2 & F3 \\
\hline 1 & 1107 & 2150 \\
2 & 1147 & 2245 \\
3 & 1198 & 2345 \\
4 & 1242 & 2431 \\
5 & 1288 & 2539 \\
6 & 1335 & 2632 \\
7 & 1374 & 2729 \\
8 & 1425 & 2809 \\
9 & 1467 & 2912 \\
\hline
\end{tabular}

Note-Steady-state $F 2=1,198 \mathrm{~Hz} ; \mathrm{F3}=2,396 \mathrm{~Hz}$.

[da] stop continuum, and a [fa]-to-[ $[\theta a]$ fricative continuum, using the same set of formant transitions, but with a uniform neutral frication at the beginning of each syllable.

Even though there is evidence (Harris, 1958) that the transitions are the primary cue to place for a choice between [fa] and $[\theta a]$, with relatively little place information in the frication, we still needed to take precautions to produce a frication that was neutral between [f] and $[\theta]$. To do this, we used information from the FOVE (Synthesis by Rule) program at Haskins (Ingemann, Note 3) and chose OVE parameters that were midway between the FOVE parameters for [f] frication and $[\theta]$ frication. The resulting frication had pole values of 1,233 and $2,540 \mathrm{~Hz}$. The peak intensity of the fricative portion was approximately $17 \mathrm{~dB}$ below that of the vowel. In addition, an aperiodic noise source excited F1, F2, and F3 at $200,1,404$, and $2,749 \mathrm{~Hz}$. The frication was $126 \mathrm{msec}$ long.

To produce a natural-sounding vocalic portion and to center our transition continuum on the labiodental-to-interdental region, we began by recording tokens of [fa] and [ $\theta \mathrm{a}]$ spoken by one of the authors (P.W.J.). We used these natural tokens as the basis for a copy synthesis: The waveform was sampled at a rate of $10 \mathrm{kHz}$ and bandpassed at $90 \mathrm{~Hz}$ to $4.9 \mathrm{kHz}$, providing input for a ubiquitous spectrum analyzer taking 128 samples per frequency span (approximately $4.9 \mathrm{kHz}$ bandwidth), repeated every $12.8 \mathrm{msec}$. The spectrum data were wideband filtered $(240 \mathrm{~Hz})$, and we used a peakextraction routine to provide values for the parameters for the OVE IIIc synthesizer.

Given parameters for the vocalic portion of [fa] and [ $\theta \mathrm{a}]$ from the copy synthesis, we constructed the rest of the transition continuum as follows: First, we took the copy synthesis versions of [fa] and [ $\theta \mathrm{a}]$ as the endpoints of a five-item continuum generated by interpolating the starting frequency values for $F 2$ (in steps of about $43 \mathrm{~Hz}$ ) and F3 (in steps of about $95 \mathrm{~Hz}$ ). Second, we extended the continuum two steps beyond each of the previous end- points; thus the copy synthesis versions of natural [fa] and [ $\theta \mathrm{a}$ ] corresponded to Stimuli 3 and 7 in the final continuum. Table 3 gives the starting frequency values for the transitions for the whole continuum. While the starting frequencies varied, all the stimuli reached steady-state values for $F 2$ of $1,198 \mathrm{~Hz}$ after a $42-\mathrm{msec}$ transition and for $F 3$ of $2,396 \mathrm{~Hz}$ after a 54-msec transition. All stimuli had the same F1 and fundamental frequency contours: F1 began at $479 \mathrm{~Hz}$ and rose to a steady-state value of $777 \mathrm{~Hz}$ after a 54-msec transition; the fundamental began at $138 \mathrm{~Hz}$, peaked $60 \mathrm{msec}$ later at $144 \mathrm{~Hz}$, and then gradually declined to $118 \mathrm{~Hz}$. The whole vocalic portion, transitions plus steady-state vowel, was 192 msec long.

Without the frication, listeners identified these stimuli as syllables beginning with voiced stops, ranging from "ba" (Stimulus 1) to "da" (Stimulus 9). When the frication was attached immediately before the transitions (giving a stimulus $318 \mathrm{msec}$ long), listeners identified the new stimuli as syllables beginning with voiceless fricatives, ranging from "fa" (Stimulus 1) to " $\theta \mathrm{a}$ " (Stimulus 9).

We prepared identification and "oddity" discrimination tapes for both continua. The identification tapes had 15 tokens of each of the nine stimuli arranged in a random order with a $3-\mathrm{sec}$ ISI. In the discrimination tapes, stimuli were presented in groups of three, with each triad composed of two tokens of one stimulus and one token of a second stimulus three steps away on the continuum, for example, Stimulus 1 vs. Stimulus 4. For each comparison, there were six possible arrangements (AAB, ABA, BAA, $\mathrm{BBA}, \mathrm{BAB}, \mathrm{ABB})$ and six possible three-step comparisons (1-4, $2-5,3-6,4-7,5-8,6-9)$; thus we have a total of 36 triads. The stimulus tapes had two tokens of each triad, giving a total of 72 stimuli, arranged in a random order with a 1-sec interval between elements of a triad and a 3-sec interval between triads.

Procedure. The subjects heard the stimuli over Koss Pro 4AAA headphones; using a sound level meter (General Radio Model 1565-A), we adjusted the volume so that the stimuli were played at a level of approximately $72 \mathrm{~dB}$ (A) SPL. The subjects listened to the stop and fricative tapes on successive days, with the order counterbalanced across subjects. On each day, they did the identification task before the discrimination task. Following their Day 2 identification and discrimination tasks, the subjects did a relabeling task in which they were required to identify stimuli from one continuum using labels appropriate to the other continuum. Thus, half the subjects heard the stop tape as a forced choice "f $f$ " vs. " $\theta$," and the other half heard the fricative tape as a forced-choice "b" vs. "d." After this relabeling task, the subjects repeated the discrimination task for the stimuli they had just relabeled. Table 4 gives the complete design of the experiment.

Instructions. For the identification tapes, subjects listening to the stop tape were told that the stimuli represented "ba" or "da" and that they should write " $b$ " or " $d$ " on their answer sheets accordingly. Similarly, subjects listening to the fricative tape were told

Table 4

Design of Experiment 2

\begin{tabular}{|c|c|c|c|}
\hline & Day 1 & Day 2 & Day 2 \\
\hline \multicolumn{4}{|c|}{ Group $1(N=5)$} \\
\hline $\begin{array}{l}\text { Identification } \\
\text { Discrimination }\end{array}$ & $\begin{array}{l}{[\mathrm{ba}]-[\mathrm{da}]} \\
{[\mathrm{ba}]-[\mathrm{da}]}\end{array}$ & $\begin{array}{l}{[\mathrm{fa}]-[\theta \mathrm{a}]} \\
{[\mathrm{fa}]-[\theta \mathrm{a}]}\end{array}$ & $\begin{array}{l}{[\mathrm{fa}]-[\theta \mathrm{a}] \text { relabeled }[\mathrm{ba}]-[\mathrm{da}]} \\
{[\mathrm{fa}]-[\theta \mathrm{a}]}\end{array}$ \\
\hline \multicolumn{4}{|c|}{ Group $2(N=5)$} \\
\hline $\begin{array}{l}\text { Identification } \\
\text { Discrimination }\end{array}$ & $\begin{array}{l}{[\mathrm{ba}]-[\mathrm{da}]} \\
{[\mathrm{ba}]-[\mathrm{da}]}\end{array}$ & $\begin{array}{l}{[\mathrm{fa}]-[\theta \mathrm{a}]} \\
{[\mathrm{fa}]-[\theta \mathrm{a}]}\end{array}$ & $\begin{array}{l}{[\mathrm{ba}]-[\mathrm{da}] \text { relabeled }[\mathrm{fa}]-[\theta \mathrm{a}]} \\
{[\mathrm{ba}]-[\mathrm{da}]}\end{array}$ \\
\hline \multicolumn{4}{|c|}{ Group $3(N=5)$} \\
\hline $\begin{array}{l}\text { Identification } \\
\text { Discrimination }\end{array}$ & $\begin{array}{l}{[\mathbf{f a}]-[\theta \mathbf{a}]} \\
{[\mathbf{f a}]-[\theta \mathrm{a}]}\end{array}$ & $\begin{array}{l}{[\mathrm{ba}]-[\mathrm{da}]} \\
{[\mathrm{ba}]-[\mathrm{da}]}\end{array}$ & $\begin{array}{l}\text { [ba] }-[\mathrm{da}] \text { relabeled }[\mathrm{fa}]-[\theta \mathrm{a}] \\
{[\mathrm{ba}]-[\mathrm{da}]}\end{array}$ \\
\hline \multicolumn{4}{|c|}{ Group $4(\mathrm{~N}=5)$} \\
\hline $\begin{array}{l}\text { Identification } \\
\text { Discrimination }\end{array}$ & $\begin{array}{l}{[\mathrm{fa}]-[\theta \mathrm{a}]} \\
{[\mathrm{fa}]-[\theta \mathrm{a}]}\end{array}$ & $\begin{array}{l}{[\mathrm{ba}]-[\mathrm{da}]} \\
{[\mathrm{ba}]-[\mathrm{da}]}\end{array}$ & $\begin{array}{l}{[\mathrm{fa}]-[\theta \mathrm{a}] \text { relabeled }[\mathrm{ba}]-[\mathrm{da}]} \\
{[\mathrm{fa}]-[\theta \mathrm{a}]}\end{array}$ \\
\hline
\end{tabular}


that the stimuli represented "fa" or "tha" and that they should write " $f$ " or " $t$ " accordingly. For the discrimination task, subjects were told that they would be hearing stimuli in groups of three, and that they were to decide which stimulus in each group differed from the other two; they were to indicate their choices by circling the appropriate letters $(A, B, C)$ on prepared answer sheets. For the relabeling task, subjects were told that we wanted them to label some of the stimuli they had already heard with new labels, so that subjects relabeling the stop tape were to identify the stimuli as "fa" or "tha" and to write " $f$ " or " $t$ " accordingly, and subjects relabeling the fricative tape were to identify the stimuli as "ba" or "da" and to write " $b$ " or " $d$ " accordingly. The subjects were told that they might find the task difficult, but that they should do the best they could. For the discrimination task that followed the relabeling task, the subjects were told that they were hearing the same stimuli again, and that they could label the stimuli in any way that helped them to do the task, using the new labels, the original appropriate labeis, both, or neither.

\section{Results}

Identification. Figure 1 gives the labeling functions for the stop and fricative continua, plotting the percentage of responses, across all subjects, that each stimulus was identified as labial (" $f$ " for the fricative continuum, " $b$ " for the stop continuum). It is clear from Figure 1 that the crossover for the fricative function is considerably further forward (further toward the labial end) than that of the stop function. This in itself suggests that the locus of the place-of-articulation boundary is different for the two series. We estimated stop and fricative boundaries for each subject by fitting a normal ogive function to his identification data (Kling \& Riggs, 1971); the two left-hand columns in Table 5 list the individual boundaries. Note that, for all 20 subjects, the fricative boundary is further forward than the stop boundary. The mean boundaries were 4.35 for the fricative continuum and $\mathbf{5 . 9 9}$ for the stop continuum; this difference is significant well beyond the $\mathrm{p}<.01$ level $[\mathrm{t}(19)=13.04]$.

Discrimination. Figure 2 gives the mean three-step discrimination functions for the stops and fricatives, along with predicted discrimination functions calculated from the identification data (Liberman, Harris, Hoffman, \& Griffith, 1957; Pollack \& Pisoni, 1971). The peak for the fricatives seems to be somewhat

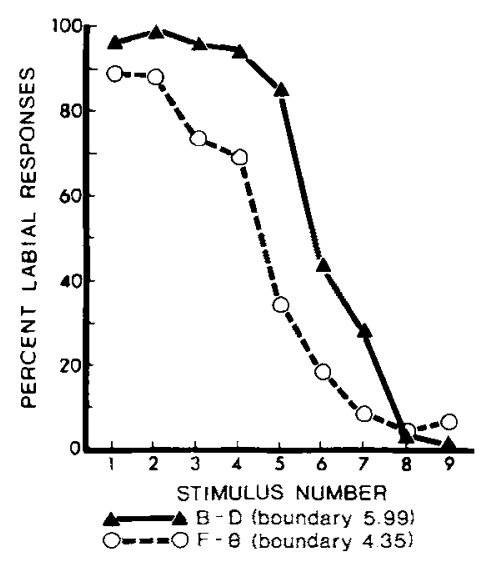

Figure 1. Labeling functions for the stop and fricative continua.
Table 5

Individual Subject's Category Boundaries for the Identification and Relabeling Conditions in Experiment 2

\begin{tabular}{|c|c|c|c|c|}
\hline \multirow{2}{*}{$\begin{array}{c}\text { Subject } \\
\text { Number }\end{array}$} & \multicolumn{2}{|c|}{ Identification } & \multicolumn{2}{|c|}{ Relabeling } \\
\hline & $b-d$ & $f-\theta$ & $f-\theta \rightarrow b-d$ & $b-d \rightarrow f-\theta$ \\
\hline $\begin{array}{r}1 \\
4 \\
5 \\
7 \\
11 \\
12 \\
13 \\
14 \\
15 \\
16\end{array}$ & $\begin{array}{l}5.19 \\
6.53 \\
4.59 \\
5.80 \\
6.27 \\
6.62 \\
6.31 \\
6.19 \\
5.43 \\
5.45\end{array}$ & $\begin{array}{l}4.18 \\
4.56 \\
3.04 \\
3.45 \\
3.53 \\
5.16 \\
4.20 \\
4.46 \\
3.90 \\
4.40\end{array}$ & $\begin{array}{l}4.27 \\
5.63 \\
4.49 \\
5.19 \\
5.39 \\
5.22 \\
5.87 \\
4.73 \\
5.00 \\
5.15\end{array}$ & \\
\hline $\begin{array}{l}\text { Mean } \\
\text { SD }\end{array}$ & $\begin{array}{r}5.84 \\
.66\end{array}$ & $\begin{array}{r}4.09 \\
.62\end{array}$ & $\begin{array}{r}5.09 \\
.49\end{array}$ & \\
\hline $\begin{array}{r}2 \\
3 \\
6 \\
8 \\
9 \\
10 \\
17 \\
18 \\
19 \\
20\end{array}$ & $\begin{array}{l}5.57 \\
6.46 \\
6.24 \\
5.22 \\
5.14 \\
5.87 \\
6.07 \\
7.79 \\
6.03 \\
7.00\end{array}$ & $\begin{array}{l}3.96 \\
4.66 \\
4.68 \\
3.39 \\
4.62 \\
4.32 \\
4.30 \\
7.26 \\
3.63 \\
5.22\end{array}$ & & $\begin{array}{l}5.10 \\
3.57 \\
5.20 \\
1.91 \\
3.55 \\
4.81 \\
4.36 \\
4.38 \\
5.71 \\
5.22\end{array}$ \\
\hline $\begin{array}{l}\text { Mean } \\
\text { SD }\end{array}$ & $\begin{array}{r}6.14 \\
.81\end{array}$ & $\begin{array}{l}4.60 \\
1.08\end{array}$ & & $\begin{array}{l}4.38 \\
1.12\end{array}$ \\
\hline $\begin{array}{l}\text { Grand Mean } \\
\text { SD }\end{array}$ & $\begin{array}{r}5.99 \\
.73\end{array}$ & $\begin{array}{r}4.35 \\
.90 \\
\end{array}$ & & \\
\hline
\end{tabular}

forward of the peak for the stops, consistent with the results for the identification task. To test this, the data were submitted to an ANOVA of a 2 (series: stop vs. fricative) by 2 (obtained vs. predicted) by 6 (stimulus comparison, e.g., 1-4, 2-5, etc.) repeated measures design. This analysis showed significant main effects for both series $[F(1,19)=20.15, p<.01]$ and stimulus comparison $[\mathrm{F}(1,19)=23.25, \mathrm{p}<.01]$. The first shows that the stops are significantly more discriminable than the fricatives, while the second shows that both discrimination functions have significant peaks. In addition, the interaction between series and stimulus comparison was also significant $[F(5,95)=23.75$, $\mathrm{p}<.01$ ], showing that the functions have different shapes, perhaps implying that the peaks occurred in different locations for the two series. There was no evidence of a significant main effect for obtained vs. predicted $[F(1,19)=2.6]$, and no interactions with this factor approached significance. This shows good agreement between the identification and discrimination data, supporting the assumption that perception of place is categorical in both stops and fricatives.

Relabeling. Subjects were instructed to identify stimuli from one series using labels appropriate to the other. Ten subjects relabeled the [ba]-[da] stimuli from the stop series as fricatives "fa" and " $\theta a$ "; the other 10 relabeled the [fa]-[0a] fricative stimuli as stops. Figure 3 shows the mean identification functions from the relabeling task for each group, together with the 


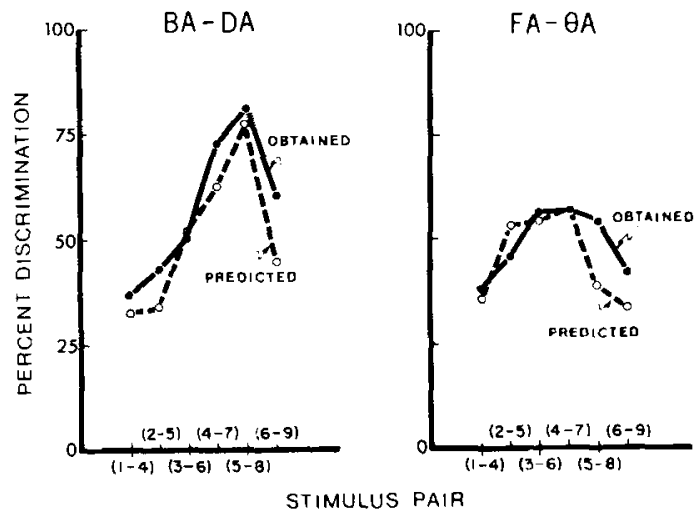

Figure 2. Discrimination functions, obtained and predicted.

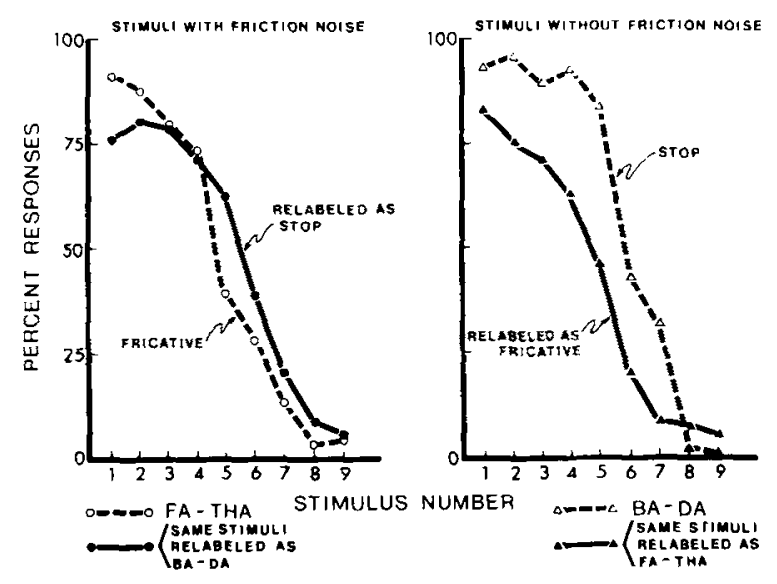

Figure 3. Relabeling funclions and original labeling functions of the same subjects for the stop and fricative continua.

mean identification functions from the same subjects' original (appropriate) labeling of the same stimuli. It is clear that the effect of the instructions in the relabeling task was to shift the identification function in the direction of the new labels, so that, for example, when the stops were relabeled as fricatives the new identification function was shifted forward, toward the location of the original fricative identification function. As before, we calculated place boundaries for the individual subjects from ogives; the individual boundaries are listed in the right-hand column of Table 5. When we compare the boundaries from the relabeling task with the boundaries from the original identification task, in which the same stimuli were identified using the acoustically appropriate labels, we see that for all 20 subjects the relabeling boundary shifts from its original location toward the boundary corresponding to the new labels. Matched-pair $t$ tests confirm that the size of the shift is significant both for fricatives relabeled as stops $[\mathrm{t}(9)=4.623, \mathrm{p}<.01]$ and for stops relabeled as fricatives $[t(9)=4.988, p<.01]$.
It is clear that the change in instructions, and so presumably a change in the manner percept, shifted the boundary in the predicted direction. In the original identification task, the stop and fricative conditions differed both in instructions and in acoustic cues to manner. Does the change of instructions by itself produce as big a boundary shift as the one we found when we compared the place boundaries for acoustically cued stops and fricatives? Here we found an interesting asymmetry: There was no significant difference between the original fricative boundary and the boundary we found when the stops were relabeled as fricatives [ $\mathrm{t}(9)=.492]$; that is, a change in the instructions alone seemed to make the acoustic stops shift all the way to the fricative boundary. But there was a significant difference between the original stop boundary and the boundary we found when the fricatives were relabeled as stops $[\mathrm{t}(9)=5.207, \mathrm{p}<.01]$. In other words, the fricatives relabeled as stops shifted in the direction of the stop boundary, but less than all the way.

Postrelabeling discrimination. Figure 4 gives the mean discrimination functions for the 10 subjects tested on each continuum after the relabeling task, compared with the original mean discrimination functions for the same subjects on the same stimuli. The peaks of the discrimination functions seem to have shifted slightly in the direction of the new labels, but the striking result is the improved discriminability of $4-7,5-8$, and 6-9 when the fricatives were relabeled as stops, and the improved discriminability of 3-6 and the reduced discriminability of 5-8 and 6-9 when the stops were relabeled as fricatives. The tendency is for discriminability to improve around the boundary that is appropriate to the new labels, and to decline when the

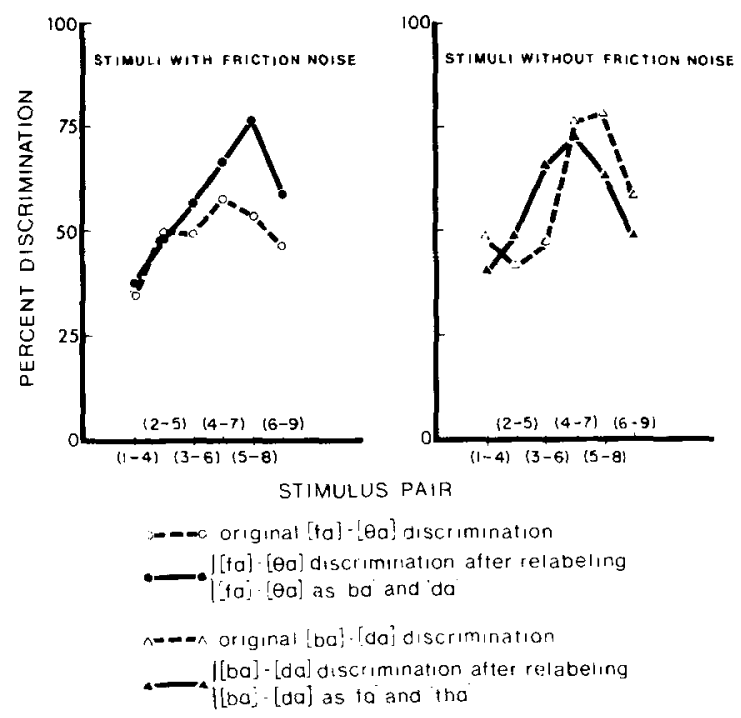

Figure 4. Postrelabeling discrimination functions and original discrimination functions for the same subjects. 
comparison crossed the old boundary but not the new. For example, 3-6 crosses the fricative boundary but not the stop boundary for the subjects who relabeled the stops as fricatives, and so discriminability improves, whereas 5-8 crosses the stop boundary but not fricative boundary, and so discriminability declines. An ANOVA of a 2 (series: stop vs. fricative) by 2 (original discrimination vs. postlabeling discrimination) by 6 (stimulus comparison) design showed a significant main effect of stimulus comparison $[F(5,90)=17.32$, $\mathrm{p}<.01]$ and a significant three-way interaction among series, original vs. postrelabeling, and stimulus comparison $[F(5,90)=4.75, p<.01]$. These results therefore show that the relabeling instructions had a significant effect on discrimination as well as identification, even though the subjects were not instructed to use the new labels for the discrimination task.

\section{Discussion}

The results from the first identification tasks show that a synthetic [ba]-[da] transition continuum has a different place boundary from the same transition continuum preceded by a [f/ $\theta$ ] synthetic frication and labeled as fricatives "fa" or " $\theta a$." The results of the first discrimination task show that the corresponding discrimination functions shift in the same way. It is not surprising to find such a place/manner dependency, given the results of earlier studies, such as Miller's (1977); our results extend these earlier studies to the limited extent that the dependency involves $[\theta]$, and that we found dependencies in discrimination as well as identification.

By themselves, the original identification and discrimination tasks do not bear on the question of phonetic processing: The differences we observe between the stop and fricative series might be caused by a dependency in phonetic processing, but they might equally well be caused by an auditory interaction between the frication and the transitions, by a trading relation between the place cue in the transitions and a place cue in the frication (if we hypothesize that our synthetic frication was a dental cue), or by a combination of all three.

We found evidence bearing on phonetic processing only when we compare the results of the original identification and discrimination tasks with the results of the relabeling task and the postrelabeling discrimination. This comparison shows that both identification and discrimination functions can be changed simply by changing the instructions. Whether or not auditory interactions played a role in producing the different place boundaries for the acoustically cued stop and fricative series, they could not have produced the contrasts in Figures 3 and 4, where we found different identification and discrimination functions for the same acoustic stimuli, depending only on the labeling instructions given to the subject.

Experiment 2 therefore confirms the main result of Experiment 1: Perceived place is dependent on labeling, and so presumably on perceived manner. Experiment 2 extends our earlier result by showing that a change in manner labeling can affect discrimination as well as identification. ${ }^{6}$ More importantly, Experiment 2 shows that the effect is phonetic rather than auditory, since a change in the instructions as to the phonetic categories to be used in classifying the stimuli is sufficient to change the categorization without a change in the stimuli themselves.

\section{GENERAL DISCUSSION}

In both of the preceding experiments, we found evidence for a dependency between perceived manner and perceived place. In Experiment 1, we constructed a stimulus that was ambiguous in manner and observed a dependency between the perceived place and manner features that we deduced from segment identifications. In Experiment 2, we manipulated perceived manner directly by changing the instructions in a forcedchoice task. Again, when the perceived manner changed, perceived place also changed. Note that this result holds for both forced-choice and free identification tasks, and for both synthetic and (modified) natural stimuli. ${ }^{7}$

We conclude that the perception of place is dependent on manner, consistent with earlier studies, such as Eimas et al.'s (1978). Our result differs from the results of these earlier studies, however, in that the shift in perceived place can occur with acoustically identical stimuli. It follows that perceived place is dependent on the (necessarily phonetic) manner percept itself rather than directly on any of the acoustic cues to manner. This result is consistent with the responseconditional model of Smith (1973), the prototype model of Oden and Massaro (1978), and the models implied by Eimas et al. (1978) and Miller (1977). It is inconsistent with any model that handles the perception of place, or dependencies between place and manner, entirely by acoustic or auditory mechanisms.

Recall that our original question was: How far can we go with nonphonetic mechanisms, mechanisms that are also used for general-purpose auditory processing? A result of the sort we describe does not, in itself, show that features are extracted using phonetic processing. The conclusion takes the form of a conditional: We make the common assumption (cf. the references cited by Miller, 1977) that feature identification is one step in the process of segment identification. If this is true, then a boundary shift for one feature, $X$, cued by the perceived value of another feature, $Y$, would indeed show that phonetic information (the perceived value of $Y$ ) was necessary input to the processing of $X$, so that phonetic processing was involved in identifying $X$. But this conclusion fails to hold if we abandon the assumption that feature identification precedes segment identification: For example, 
if we imagined that the set $[b, d, f, \theta]$ simply differed holistically, with no place or manner features involved in segment identification, then our results would show that $[\theta]$ was more similar to [b] than it was to [d], a conclusion that would (absent features) have no theoretical implications. Phonetic processing would be involved, but only in the uninteresting sense that the process produces an overtly phonetic output, the identified segment.

Consider how our result could be handled in a prototype model like that of Oden and Massaro (1978): Here a feature evaluation mechanism, which, in principle, could be a general-purpose, nonphonetic mechanism, returns not one of a set of discrete features values ("labial" or "stop"), but a point on a continuous scale ("n front" or "m continuant"). The resulting set of values would be compared to prototypes for the various segments, with appropriate weightings for different values along each dimension. Such a model would recover segments without ever recovering features in the usual sense. It would duplicate the effect of our two-boundary hypothesis by setting the "front" values differently for the stop and fricative prototypes, which, indeed, is what one would expect, given the articulation. If we are to reject a prototype model as an adequate explanation for our results, it must be for reasons independent of the data reported in this paper, which are equally consistent with the prototype model and the two-boundary model we favor.

\section{The Two-Boundary Hypothesis}

Our results show that perceived place is dependent on perceived manner, but they do not provide direct evidence about what produces this dependency. It seems clear that the perception of one or more of the cues to place is affected by perceived manner, but there are several imaginable cues that might be involved.

The most natural interpretation, and the one we at present favor, is that perceived manner affects the interpretation of the formant-transition cue. In Experiment 2 , the synthetic stimuli in a given continuum were identical except for the formant transitions, and it is therefore natural to assume that the place judgments were cued solely or primarily by the transitions. If this is so, the results of Experiment 2 would be accounted for by what we could call the "two-boundary hypothesis": Suppose that the listener can process an incoming transition using either of two boundaries. When he has identified the manner as stop, he uses one boundary; when he has identified the manner as fricative, he uses the other, which is slightly further forward (toward the labial end of the continuum), as implied by the boundaries listed in Table 5 .

How would we apply this two-boundary hypothesis to the results of Experiment 1, in which we were dealing with truncated natural syllables rather than with a synthetic continuum? Let us represent a transition continuum like the synthetic one schematically by a

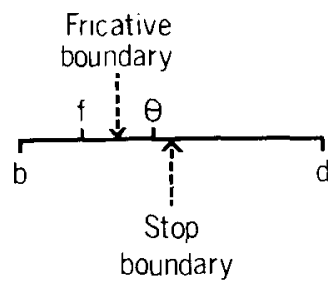

Figure 5. Schematic representation of transition continuum.

line (Figure 5); we could think of this line as representing a perceptual transition space, or, more concretely, as representing the range of formant-transition starting frequencies for some given vowel. Recall that the naturally produced stops $[b, d]$ differ in place of articulation from the corresponding fricatives $[f, v$, $\theta, \partial]$. The endpoints of the line in Figure 5 correspond to the transitions produced by bilabials [b] and alveolars [d]. Labiodentals $[f, v]$ and interdentals $[\theta, \partial]$ have intermediate places of articulation, and therefore presumably produce intermediate transitions. The fricative boundary must fall between the points corresponding to the natural labiodental and interdental transitions; Experiment 2 shows that the stop boundary must fall further back. We hypothesize that the stop boundary falls on or behind the point corresponding to the natural interdental articulation, as sketched in Figure 5. This hypothesis will account for the data of Experiment 1 as follows: When the ambiguous [ $\theta \mathrm{a}]-$ stimulus is identified as a fricative in Experiment 1, the subject uses the fricative boundary to evaluate the transition cue, and so gets the same labial/dental distribution that he got for untruncated $[\theta a]$. When $[\theta a]-$ is identified as a stop, the subject uses the stop boundary. Since the transitions produced by the $[\theta \mathrm{a}]$ articulation fall, on the average, slightly on the labial side of the stop boundary, the subject identifies the syllable mostly as labial "ba" rather than dental "da."'

\section{Alternative Hypotheses}

While we feel that this two-boundary hypothesis gives the most promising account of our data, our results are also consistent with the hypothesis that there is a single perceptual boundary for the transition cue and that the shift in place identifications (and so the shift in the apparent boundary in the synthetic transition continuum) results from a trading relation between the transition cue and some other place cue, $X$. In such an analysis, it would be the perception of cue $X$ that was dependent on perceived manner and so was crucially phonetic. In other words, our results show that the perception of some place cue is sensitive to perceived manner, but they do not show that the relevant cue is necessarily the transition cue itself.

While we have not done any direct investigation of alternative cues, at least two candidates for cue $\mathrm{X}$ are imaginable. (1) Our stops consistently lacked bursts; [b] bursts are typically smaller in amplitude than [d] 
bursts. Lack of burst might therefore be a labial cue, and it would be natural for such a cue to be relevant only when the perceived manner was stop. (2) The truncated fricatives in Experiment 1 and the stops relabeled as fricatives in Experiment 2 all lacked frication. $[\theta, \partial]$, for some speakers, have frication the length of stop bursts, markedly shorter than $[f, v]$ or other fricatives. Length of frication might therefore be a place cue for the choice $[f, v]$ vs. $[\theta, g]$, with short or zero frication being a cue for dental. Again, it would be reasonable for such a cue to be relevant only when the perceived manner was fricative.

\section{The Place Manuer Dependencies} with [f], [v], and [ $\delta$ ]

Our experiments and discussion have focused on the place/manner dependency with [ $\theta \mathrm{a}]$, but we must also consider how to account for the smaller dependencies we saw for the other truncated fricatives in Experiment 1 . We have little to say about [fa]- and [va]-; we suspect that the shift may be caused by a trading relation between the transition cue and some other place cue introduced by the truncation operation, perhaps the lack of burst or the short-frication cue discussed above. But we have no evidence bearing on this issue. The case of the voiced interdental [ $\delta$ a]-, however, requires some discussion: Taken by itself, the twoboundary hypothesis implies that the voiced and voiceless interdentals should act alike. Recall, however, that, while the voiced $\left[\gamma_{\mathrm{a}}\right]$ - showed an apparent place/ manner dependency, the size of the effect was much smaller for [ $\partial \mathrm{a}]$ - than for $[\theta \mathrm{a}]-$. When [ $\delta \mathrm{a}]-$ was identified as a stop, the identifications were only $20 \%$ labial "ba," contrasting with $75 \%$ "ba" identifications for

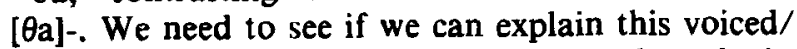
voiceless contrast within the two-boundary hypothesis. One possibility is that the perception of the transition cue might be dependent on voicing as well as manner, as suggested by the results of Eimas et al. (1978) and Miller (1977). A second possibility, perhaps sufficient in itself to explain our data, is that [g] may be articulated slightly further back than $[\theta]$, so that the transitions in [ $\gamma \mathrm{a}$ ] - fall slightly on the dental side of the stop boundary. While textbooks do not report any difference in position of articulation between $[\theta]$ and $[\gamma]$, an informal survey of phonetically trained native speakers of English showed general agreement that $[\delta]$ is articulated slightly further back than $[\theta]$, consistent with the perceptual contrast we found in Experiment 1.

\section{Conclusion}

We find the alternative hypotheses implausible, but our primary reason for preferring the two-boundary hypothesis is that it offers a possible explanation for the existence of this place/manner dependency: A boundary located at the perceptual midpoint between typical bilabial transitions and typical alveolar transi- tions would not be expected to coincide with a boundary located at the perceptual midpoint between labiodental and interdental transitions. On this view, the difference in place of articulation in production is the reason for the different boundaries in perception. This rationalization applies only if it is the transition cue that is involved; if it was lack of burst or length of frication that was crucial, then we would expect the perceptual dependency to be independent of the articulatory place/ manner dependency we have in English. The validity of this production-based explanation for the perceptual dependency can therefore be tested directly by investigating the perceptual dependency in languages that lack the English articulatory dependency.9

While we have not been able to reach a firm conclusion about which place cue is dependent on the manner percept, we wish to emphasize that our major theoretical conclusion is independent of which cue is involved:

The results of both our experiments agree in showing that the processing of the place feature will have to be sensitive to perceived manner rather than directly to any combination of the acoustic cues to manner. Because the shift occurs even when the stop and fricative stimuli are acoustically identical, in principle no purely auditory mechanism can do the job. We must postulate that phonetic processing is involved at some level in the perception of the place feature.

\section{REFERENCE NOTES}

1. Bailey, P. J., Summerfield, Q., \& Dorman, M. On the identification of sine-wave analogues of certain speech sounds (Status Report on Speech Research, SR51/52, 1-25). New Haven, Coni1: Haskins Laboratories, 1977.

2. Best, C. T., Morrongiello, B., \& Robson, R. The perceptual equivalence of two acoustic cues for a speech contrast is specific to phonetic perception (Status Report on Speech Research, SR62, 1-45). New Haven, Conn: Haskins Laboratories, 1980.

3. Ingemann, F. Speech synthesis by rule using the FOVE program (Status Report on Speech Research, SR54, 165-173). New Haven, Conn: Haskins Laboratories, 1978.

\section{REFERENCES}

Cooper, F. S., \& Mattingly, I. G. A computer-controlled PCM system for the investigation of dichotic speech perception. Journal of the Acoustical Society of America, 1969, 46, 115 (A).

Dorman, M. F. On the identification of sine-wave analogues of $\mathrm{CV}$ syllables. In E. Fischer-Jorgensen \& N. Thorsen (Eds.), Proceedings of the Ninth International Congress of Phonetic Sciences (Vol. 2). Copenhagen: Institute of Phonetics, 1980.

Emas, P. D. Tarter, V. C., Miller, J. L., \& Keuthen, N. J. Asymmetric dependencies in processing phonetic features. Perception \& Psychophysics, 1978, 23, 12-20.

Harris, K. S. Cues for the discrimination of American English fricatives in spoken syllables. Language \& Speech, 1958, 1, 1-7.

Isenberg, D., \& Liberman, A. M. Speech and non-speech percepts from the same sound. Journal of the Acoustical Society of America, 1978, 64, Suppl. No. 1 J 20.

Kı. ING, J. W., \& Riggs, L. A. (Eds.), Woodworth and Schlosberg's experimental psychology. New York: Holt, Rinehart \& Winston, 1971.

Liberman, A. M. Duplex perception and integration of cues: 
Evidence that speech is different from non-speech and similar to language. In E. Fischer-Jorgensen \& N. Thorsen (Eds.), Proceedings of the Ninth International Congress of Phonetic Sciences (Vol. 2). Copenhagen: Institute of Phonetics, 1980. Liberman, A. M., Harris, K. S., Hoffman, H. S., \& Griffith, B. C. The discrimination of speech sounds within and across phoneme boundaries. Journal of Experimental Psychology, $1957,54,358-368$.

Liberman, A. M., \& Studdert-Kennedy, M. Phonetic perception. In R. Held, H. Leibowitz, \& H. L. Teuber (Eds.), Handbook of sensory physiology: Perception. New York: SpringerVerlag, 1978.

MiLle R, J. L. Nonindependence of feature processing in initial consonants. Journal of Speech \& Hearing Research, 1977, 20, $510-518$

Oden. G. C.. \& Massaro. D. W. Integration of featural informalion in speech perception. Psychological Review, 1978, 85, 172-191.

Pollack, I., \& Pisoni, D. B. On the comparison between identification and discrimination tests in speech perception. Psychonomic Science, 1971, 24, 299-300.

Pisoni, D. B. Some remarks on the perception of speech and nonspeech signals. In E. Fischer-Jorgensen \& N. Thorsen (Eds.), Proceedings of the Ninth International Congress of Phonetic Sciences (Vol. 3). Copenhagen: Institute of Phonetics, 1980.

Sмıтн, P. T. Feature-testing models and their application 10 perception and memory for speech. Quarterly Journal of EXperimental Psvchology, 1973, 25, 511-534.

Stevens, K. N.. \& Blumstein. S. E. Invariant cues for place of articulation in stop consonants. Journal of the Acoustical Society of America, 1978, 64, 1358-1368.

\section{NOTES}

1. In accordance with common linguistic practice, we will often use the cover term "labial" to refer to both bilabials $[p, b, m]$ and labiodentals [f,v] and the cover term "dental" to refer to both alveolars $[t, d, n]$ and interdentals $[\theta, \delta]$. Underlying this choice of terminology is the assumption that, at the level of phonological structure, bilabials and labiodentals have the same abstract feature(s) for place of articulation, as do alveolars and interdentals.

2. We selected voiced rather than voiceless stops for the comparison because preliminary results showed that truncated fricatives, whether voiced or voiceless, were usually perceived as voiced stops.

3. This truncation operation limited our choice of speakers, since for some speakers the friction overlaps with the vocalic part to the extent that a cut after the friction eliminates so much of the transitions that no initial consonant is heard, while a cut at the beginning of the vocalic part leaves so much friction that the initial consonant is unambiguously fricative. The tokens for this experiment were chosen from those speakers for whom a cut point at the beginning of the vocalic portion (and so presumably including all the voiced transitions) gave a reasonably stop-like percept.
4. As we had expected, the resuits for the interdentals showed substantial variation from speaker to speaker. For two speakers, T.H. and P.N., [ $\theta \mathrm{a}]-$, when perceived as a stop, was primarily identified as labial "ba" (98\% "ba" responses for T.H., $91 \%$ for P.N.). For the other two, it was balanced on the labial/dental border (D.l.: 55\%" "ba," 45\%" "da"; P.B.: $42 \%$ "ba," 58\%" "da"). For all four speakers, however, [ $\theta$ a]- received significantly more labial responses when it was identified as a stop than when it was identified as a fricative ( $p<.01$ by chi-square in each case, pooling results for all subjects). Recall that T.H. and D.I. are American, while P.N. and P.B. are English. This suggests that the interspeaker variation we see here reflects individual differences rather than regional dialects.

5. We can test this by looking at the responses of individual subjects to individual tokens. Each subject heard 10 repetitions of each token. Suppose we score only those cases in which a subject gave at least two stops and two fricative identifications. The resulting pattern, both for the pooled data and for the individual speakers,

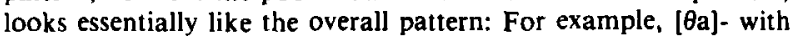
stop manner was identified as labial $69 \%$ of the time and as dental, $31 \%$; with fricative manner, it was labial $26 \%$ and dental $74 \%$, This difference is significant ( $p<.01$ by chi-square for the pooled data; $p<.025$ by chi-square for each of the speakers independently).

6. At the suggestion of David Pisoni, this experiment was replicated in its entirety using fewer subjects (eight) but more observations per data point ( 30 instead of 15 for identification, 24 instead of 12 for discrimination) for each subject. The main results of the replication were in accordance with those presented here.

7. Note that our stimuli are all [Ca] syllables. Preliminary results suggest that the effect we have observed is sensitive to both vowel height and rounding, and to CV vs. VC order.

8. One discrepancy requires comment: Recall that the identification data from Experiment 2 implied a fricative boundary about 4.35 and a stop boundary about 5.99 . This would imply that natural $[\theta]$ transitions should correspond to a point on the synthetic continuum around Stimulus 5, which does give identification data similar to natural [Ba]-. However, the copy-synthesis version of P.W.J.'s natural $[\theta a]$ is Stimulus 7 . Given the small absolute difference between Stimuii 5 and 7 on the synthetic continuum $(e .8 ., 86 \mathrm{~Hz}$ in the starting frequency of $F 2$ ), the lack of precision in copy synthesis, and the different general character of natural and synthetic stimuli, we do nol believe that this discrepancy is evidence against the twoboundary hypothesis.

9. It is important to notice that this rationalization for our apparent boundary shift cannot be extended to the boundary shifts found by Miller (1977): [p,b,m] are all equally bilabial, and $[t, d, n]$ equaliy alveolar, so there is no apparent manner/place interaction in articulation to appeal to. It will therefore be important to test whether the effect Miller found works in the same way as ours, in particular whether we can replicate Miller's shifts by manipulating the manner percept while holding the acoustic stimulus constant.

(Received for publication June 2, 1980; accepted revision received October 22,1980 .) 